\title{
Epidemiología de la neutropenia febril en pacientes adultos con neoplasia hematológica, en un período de 26 meses en el Hospital Pablo Tobón Uribe, Colombia
}

\author{
Camilo Madrid, Laura Díaz, Juan Combariza, Kenny Gálvez, \\ Viviana Olaya, Isabel Ramírez y Jorge Donado
}

\section{Epidemiology of febrile neutropenia in adult patients with hematologic neoplasms in a period of 26 months in Hospital Pablo Tobón Uribe, Colombia}

Background: Febrile neutropenia (FN) is a significant adverse effect post-chemotherapy due to its high morbidity and mortality. There are few studies in our country with these kind of patients. Objective: To describe the characteristics and mortality in patients with hematologic neoplasms who developed FN post-chemotherapy. Methodology: A descriptive case series in patients with hematologic neoplasms who developed FN post-chemotherapy in Hospital Pablo Tobón Uribe. Results: 101 episodes of FN in 43 patients. The median age was 44 years. $63.5 \%$ of patients had no apparent clinical focus of infection at admission, $11.8 \%$ had soft tissue compromise and $8.9 \%$ urinary tract infection. Bacteremia was documented in $41.5 \%$ and catheter-associated bacteremia in 3.9\%. The most common organisms were Escherichia coli 43.4\%, Klebsiella pneumoniae 17.3\% and Staphylococcus aureus $8.6 \%$. Of those isolated in blood $84.7 \%$ were Gram negative rods and $15.2 \%$ were Gram positive bacteria. Piperacillin/tazobactam was the most common empirically prescribed antibiotic (81.1\%). Mortality of FN episodes occurred in $8(7.92 \%)$ patients, $5(62.5 \%)$ attributable to infection and $3(37.5 \%)$ due to progression of hematologic malignancy with a resolution of FN. Conclusions: In our case series of FN the microbiological characteristics differed significantly from developed countries, but a similar mortality rate per episode was observed.

Key words: Hematologic neoplasms, neutropenia, fever, bacteremia.

Palabras clave: Neoplasias hematológicas, neutropenia, fiebre, bacteriemia.

\section{Introducción}

$\mathrm{L}$ a neutropenia febril (NF) y sus complicaciones infecciosas son algunos de los efectos adversos más importantes derivados del manejo del paciente hematológico con enfermedad maligna en tratamiento con quimioterapia. La NF debe ser entendida, hasta que se demuestre lo contrario, como de causa infecciosa en un hospedero inmunocomprometido (enfermedad de base y tratamiento con citostáticos) y debe abordarse como una emergencia médica por la alta morbilidad y mortalidad que conlleva; en la actualidad, dependiendo de la serie estudiada, la mortalidad atribuible en los pacientes hematológicos oscila entre 4 y $9 \%{ }^{1}$.

Según cuál sea la enfermedad de base, el estado inmunológico, las co-morbilidades y el tipo de intervención (esquema de quimioterapia, toxicidad hematológica intrínseca, dosis y duración), entre otras características, se modifica el riesgo de desarrollar NF, su gravedad y finalmente su pronóstico. En la actualidad, se estima que, de manera global, 60 a $85 \%$ de los adultos con cáncer hematológico y quimioterapia desarrollarán una $\mathrm{NF}^{2-4}$.

Conociendo el impacto de la NF en la mortalidad de los pacientes, es claro desde los años 60' que se debe iniciar de manera precoz antibioticoterapia de amplio espectro; un retraso en su inicio se relaciona con una mortalidad de hasta $70 \%$ como se documentó en estudios iniciales ${ }^{5-7}$.

Hoy en día se recomienda iniciar monoterapia con antimicrobianos de amplio espectro que garanticen el tratamiento adecuado de Pseudomonas aeruginosa como son cefepime, meropenem, imipenem o piperacilina/ tazobactam ${ }^{8}$, sin haberse demostrado hasta el momento superioridad en ninguno de ellos ${ }^{9-11}$.

La presencia de escasos datos epidemiológicos en nuestro medio de los pacientes con enfermedad hematológica maligna que desarrollan NF post-quimioterapia, podría afectar las decisiones clínicas en nuestra práctica médica, razón por la cual el grupo de Hematología del Hospital Pablo Tobón Uribe (HPTU) decidió realizar un estudio para conocer las características demográficas, microbiológicas, clínicas y los desenlaces de los pacientes descritos con el objetivo de conocer cuál o cuáles enfermedades hematológicas, esquemas citostáticos, características clínicas, hallazgos microbiológicos y desenlaces fueron los que predominaron en la muestra estudiada.

Es importante identificar si existe o no en nuestro
Universidad Pontificia Bolivariana. Medellín, Colombia. Facultad de Medicina. Residente de Medicina Interna (CM, LD).

Departamento de Epidemiología (JD).

Hospital Pablo Tobón Uribe. Medellín, Colombia.

Unidad de Hematología (LD, JC, $K G, V O)$.

Departamento de Infectología (IR). Departamento de Epidemiología (JD).

Universidad de Antioquia. Medellín, Colombia. Departamento de Medicina Interna, sección de Enfermedades Infecciosas (IR)

Los autores declaran que no tienen conflictos de intereses ni recibieron dinero de institución alguna.

Recibido: 14 de mayo de 2012 Aceptado: 4 de febrero de 2013

Correspondencia a:

Camilo Alberto Madrid Muñoz. camilomadrid@yahoo.com 
medio, alguna diferencia con respecto a los otros estudios descritos en la literatura científica (nacional e internacional), que lleve a plantear ajustes en el enfoque terapéutico empírico y dirigido de estos pacientes, como predecir la aparición de NF, iniciar un tratamiento y/o profilaxis oportunos y adecuados.

\section{Metodología}

Estudio observacional, descriptivo, de serie de casos, de pacientes adultos con enfermedad hematológica maligna que desarrollaron NF post-quimioterapia y recibieron manejo hospitalario en el HPTU, en un período de tiempo de 26 meses.

El HPTU es una fundación privada sin ánimo de lucro, localizado en la ciudad de Medellín, Antioquia, Colombia. Es un hospital de carácter general, con alto nivel de complejidad, que cuenta con 371 camas de hospitalización entre los servicios de hospitalización general, unidad de cuidados especiales y unidad de cuidado intensivo adulto y pediátrico, incluyendo cinco camas en la Unidad de Trasplante de Progenitores Hematopoyéticos. El índice ocupacional ha superado al $90 \%$ en los últimos cinco años.

\section{Población}

Pacientes $>15$ años, sin discriminación de sexo, con enfermedad hematológica, que recibieron tratamiento con quimioterapia y desarrollaron NF entre los años 2009 y 2011, y fueron manejados acorde al protocolo institucional de NF (Anexo 1). Se excluyeron aquellos pacientes que no tenían en la historia clínica la información de las variables demográficas, clínicas, microbiológicas y desenlaces durante la hospitalización por episodio de NF.

Los casos de NF se definieron acorde a los criterios indicados en las guías de la $\operatorname{IDSA}^{8}$ y $\mathrm{NCCN}^{12}$ :

Fiebre: Temperatura oral única $\geq 38,3^{\circ} \mathrm{C}\left(101^{\circ} \mathrm{F}\right)$ o temperatura oral $\geq 38,0^{\circ} \mathrm{C}\left(100.4^{\circ} \mathrm{F}\right)$ durante un período sostenido de una hora

Neutropenia: Recuento de neutrófilos $<500$ céls $/ \mathrm{mm}^{3}$ o recuento de neutrófilos que se presuma disminuya a $<500$ céls $/ \mathrm{mm}^{3}$ en las siguientes $48 \mathrm{~h}$. Se definió neutropenia profunda como recuento de neutrófilos $<100$ céls $/ \mathrm{mm}^{38}$.

Se definió bacteriemia asociada al catéter acorde a las guías de la IDSA $2009^{13}$.

La mortalidad atribuible a infección se realizó con la verificación de la historia clínica por dos investigadores, quienes, de manera independiente, evaluaron las historias para calificar la relación del deceso con la infección durante el episodio de NF.

\section{Recolección de datos}

Los pacientes con diagnóstico de NF fueron identificados a través de la base de datos del grupo de Hematología del HPTU. Se evaluó la historia clínica de cada uno de ellos para identificar las variables (demográficas, clínicas, microbiológicas y desenlace) de cada caso en particular. Se analizaron los datos por episodio y no por paciente, lo cual quiere decir que si un paciente tenía varios episodios de NF, cada uno de ellos se evaluaría como un episodio diferente.

La identificación y las pruebas de sensibilidad fueron realizadas por el laboratorio de microbiología usando el sistema VITEK ${ }^{\circledR} 2$ Compact (bioMérieux, Lyon, Francia), tarjetas AST-P577.

\section{Análisis estadístico}

Las variables cuantitativas se expresaran como mediana y rango intercuartílico (RIQ) $\left(\mathrm{P}_{25-75}\right)$. Las variables cualitativas como frecuencias absolutas y relativas. Se utilizó el programa Microsoft Office Excel 2010.

La conducción de esta serie de casos fue orientada según las recomendaciones de Dekkers et $\mathrm{al}^{14}$.

El protocolo de investigación fue aprobado por el Comité de Investigaciones y Ética en Investigaciones del Hospital Pablo Tobón Uribe.

\section{Resultados}

Se identificaron 101 episodios de NF que cumplían con los criterios de inclusión y la definición de las guías descritas, en un total de 43 pacientes. No se excluyeron pacientes porque se logró identificar la información de todas las variables.

La mediana de la edad fue de 44 años RIQ (27 a 51,5). La razón hombre-mujer fue de 1:1, la mediana de neutrófilos al momento del diagnóstico fue de 100 RIQ (30 a 200); 48 (47,5\%) episodios al momento del diagnóstico cumplían con la definición de neutropenia profunda y la mediana de días de neutropenia fue de 11 RIQ (7 a 20).

Los tres diagnósticos hematológicos más frecuentes fueron leucemia mieloide aguda-LMA (n: 18; 41,9\%), leucemia linfoide aguda-LLA (n: $10 ; 23,3 \%)$ y linfoma $\mathrm{B}$ difuso de células grandes-LBDCG (n: 5; 11,6\%). (Tabla 1).

Los tres esquemas de quimioterapia más frecuentes fueron dosis altas de citarabina (HIDAC) (n: 21; 20,8\%), HYPERCVAD (n: 18; 17,8\%) y citarabina más idarrubicina $(7 \times 3)(n: 15 ; 14,8 \%)$ (Tabla 2).

Se presentaron siete episodios en dos pacientes con infección por VIH, uno de ellos con diagnóstico de linfoma de Burkitt y el otro con diagnóstico de LBDCG asociado a VIH.

\section{Expresión clínica}

Se evaluaron los posibles focos infecciosos de los 101 episodios estando ausentes en la mayoría (n: 64; 63,5\%). Se detectaron infección de tejidos blandos (n: 12; 11,8\%), 
foco urinario (n: 9; 8,9\%) y colitis neutropénica (n: 6; 5,9\%) (Tabla 3).

\section{Accesos vasculares}

Se registraron los accesos vasculares instalados previamente al episodios de NF; excluyendo los catéteres periféricos que se encontraban en la gran mayoría de los

Tabla 1. Diagnóstico hematológico previo al episodio de neutropenia febril en 43 pacientes. HPTU 2009-2011

\begin{tabular}{|c|c|}
\hline Enfermedad hematológica & n $(\%)$ \\
\hline Leucemia mieloide aguda & $18(41,8)$ \\
\hline Leucemia linfoide aguda & $10(23,3)$ \\
\hline LBDCG* & $5(11,6)$ \\
\hline Linfoma de Burkitt & $4 \quad(9,3)$ \\
\hline Linfoma del manto & $1 \quad(2,3)$ \\
\hline Mielofibrosis & $1 \quad(2,3)$ \\
\hline Leucemia prolinfocítica T & $1 \quad(2,3)$ \\
\hline Mieloma múltiple & $1 \quad(2,3)$ \\
\hline LBDCG asociado a $\mathrm{VIH}^{+}$ & $1 \quad(2,3)$ \\
\hline Linfoma linfoblástico & $(2,3)$ \\
\hline
\end{tabular}

Tabla 2. Quimioterapia administrada previa al episodio de neutropenia febril. HPTU 2009-2011

\begin{tabular}{|c|c|c|}
\hline Quimioterapia & & (\%) \\
\hline HIDAC* & 21 & $(20,8)$ \\
\hline HYPERCVAD ${ }^{\dagger}$ & 18 & $(17,8)$ \\
\hline $7 \times 3^{\ddagger}$ & 15 & $(14,8)$ \\
\hline IDA FLAG' & & $(8,9)$ \\
\hline $\mathrm{RCHOP**}$ & 9 & $(8,9)$ \\
\hline FRALLE $^{\dagger+}$ & 6 & $(5,9)$ \\
\hline PETHEMA & 5 & $(4,9)$ \\
\hline CODOX-M" & 5 & $(4,9)$ \\
\hline Trasplante alogénico & 4 & $(3,9)$ \\
\hline IVAC*** & 3 & $(2,9)$ \\
\hline Otros & 6 & $(5,9)$ \\
\hline \multicolumn{3}{|c|}{$\begin{array}{l}\text { *HIDAC: dosis altas de citarabina. }{ }^{+H Y P E R C V A D: ~ c i c l o f o s f a m i d a ~ h i-~} \\
\text { perfraccionada, vincristina, doxorrubicina y dexametasona y/o dosis } \\
\text { altas de metotrexate y dosis altas de citarabina. }{ }^{*} 7 \times 3 \text { : citarabina } \\
\text { e idarrubicina. "IDA FLAG: idarrubicina, citarabina y fludarabina. } \\
{ }^{* * R C H O P: ~ r i t u x i m a b, ~ c i c l o f o s f a m i d a, ~ d o x o r r u b i c i n a, ~ v i n c r i s t i n a ~ y ~} \\
\text { prednisona. }{ }^{+} \text {FRALLE: prednisona, vincristina, daunorrubicina y } \\
\text { asparaginasa. }{ }^{*} \text { yETHEMA: idarrubicina, ácido todo-transretinoico. } \\
\text { "CODOX-M: ciclofosfamida, vincristina, doxorrubicina y dosis altas } \\
\text { de metotrexate. }{ }^{* * * I V A C: ~ i f o s f a m i d a, ~ e t o p o ́ s i d o ~ y ~ d o s i s ~ a l t a s ~ d e ~} \\
\text { citarabina. }\end{array}$} \\
\hline
\end{tabular}

pacientes, 49 (48,5\%) tenían catéter central de inserción periférica PICC, 15 (14,8\%) tenían catéter venoso central CVC y 4 (3,9\%) catéter con reservorio subcutáneo.

\section{Hallazgos microbiológicos}

Se documentó bacteriemia aislada en 42 (41,6\%), bacteriemia asociada al catéter en 4 (3,9\%), colonización del catéter en 5 (4,9\%), aislamiento sólo en urocultivo en $6(5,9 \%)$ y sin ningún tipo de aislamiento en $44(43,6 \%)$ pacientes.

Los microorganismos más frecuentemente aislados en sangre (bacteriemia y bacteriemia asociada al catéter) fueron Escherichia coli (n: 20; 43,4\%), Klebsiella pneumoniae (n: 8; 17,4\%) y Staphylococcus aureus (n: 4; $8,7 \%$ ). Infecciones polimicrobianas hubo en 8 episodios $(17,4 \%)$ (Tabla 4).

De los 46 episodios con bacteriemias, incluyendo las polimicrobianas, $39(84,8 \%)$ fueron por bacilos gramnegativos y $7(15,2 \%)$ por cocáceas grampositivas.

Respecto a las infecciones polimicrobianas en sangre, se presentaron las siguientes combinaciones: tres casos de $E$. coli $+K$. pneumoniae y un caso de cada una de las siguientes combinaciones: K. pneumoniae + Pseudomo-

Tabla 3. Probable foco infeccioso clínico al momento del diagnóstico de neutropenia febril. HPTU 2009-2011

\begin{tabular}{|lrc|}
\hline Foco infeccioso & $\mathbf{n}(\mathbf{( \% )}$ \\
Tejidos blandos & $12(11,8)$ \\
Urinario & $9(8,9)$ \\
Colitis & $6(5,9)$ \\
Pulmón & $5 \quad(4,9)$ \\
Pulmón y urinario & $2(1,9)$ \\
Colitis y urinario & $1(0,9)$ \\
Tejidos blandos y urinario & $1(0,9)$ \\
Tejidos blandos, pulmón y colitis & $1(0,9)$ \\
\hline
\end{tabular}

Tabla 4. Identificación microbiológica en bacteriemia y bacteriemia asociada al catéter. HPTU 2009-2011

\begin{tabular}{|lr|} 
Microorganismo & $\mathbf{n}(\mathbf{\%})$ \\
\hline E. coli & $20(43,4)$ \\
K. pneumoniae & $8(17,3)$ \\
Polimicrobiano & $8(17,3)$ \\
S. aureus & $4(8,6)$ \\
P. aeruginosa & $2(4,3)$ \\
K. oxytoca & $1(2,1)$ \\
S. marcescens & $1(2,1)$ \\
Streptococcus spp & $1(2,1)$ \\
Streptococcus agalactiae & $1(2,1)$ \\
\hline
\end{tabular}


nas aeruginosa; Enterobacter cloacae + Enterobacter asburiae; Staphylococcus coagulasa negativa + Streptococcus mitis; Stenotrophomonas maltophilia + Fusarium sp y K. pneumoniae + E. cloacae + Candida tropicalis.

Sólo se documentó un episodio de tuberculosis pleural en un paciente de 42 años con linfoma de Burkitt que, durante un episodio de NF, se encontró en la radiografía de tórax una efusión pleural y en el cultivo de líquido pleural se obtuvo crecimiento de Mycobacterium tuberculosis, evolucionando favorablemente con la terapia antituberculosa.

Hubo dos episodios de fungemia por Candida tropicalis y Fusarium sp, en ambos casos en el contexto de una infección polimicrobiana como se describió anteriormente, y el primero de ellos con desenlace fatal. Se identificaron cinco casos probables de aspergilosis pulmonar sin lograrse la confirmación microbiológica. Los criterios diagnósticos empleados fueron presencia de nódulos en estudios imagenológicos de tórax y/o galactomanano positivo en sangre; todos los casos tenían bacteriemia confirmada y tres de ellos fallecieron (dos casos atribuibles a la infección y uno por progresión de la neoplasia hematológica).

La antibioticoterapia empírica inicial utilizada en nuestra institución fue piperacilina/tazobactam en 82 $(81,2 \%)$ pacientes, seguida por meropenem en $12(11,9 \%)$. La mediana de duración fue de 12 días RIQ (7 a 14). Esta antibioticoterapia empírica se cambió en 52 pacientes $(51,5 \%)$ por las siguientes razones: en 21 casos $(40,4 \%)$ por ausencia de respuesta clínica, en $17(32,7 \%)$ por deescalamiento de la terapia, en $11(21 \%)$ por resistencia a la terapia empírica inicial, en $2(3,8 \%)$ por toxicidad y en uno $(1,9 \%)$ por ausencia de respuesta microbiológica.

Sólo cuatro episodios fueron precedidos por profilaxis con quinolonas, eran pacientes con quimioterapia previa a trasplante alogénico de precursores hematopoyéticos.

Recibieron tratamiento empírico para $S$. aureus resistente a meticilina $53(52,4 \%)$ episodios, $98,1 \%$ con vancomicina y $1,9 \%$ con linezolid; la mediana de duración fue de 5 días RIQ (3 a 8). En la mayoría de casos no se encontró en la historia clínica consignada la justificación para el inicio de esta terapia, 15/53 (28,3\%) no habían tenido una respuesta clínica adecuada al manejo empírico de primera línea.

Recibieron manejo con antifúngicos 33 (32,7\%) episodios (18 pacientes). 45,4\% con voriconazol, 18,2\% anfotericina B deoxicolato, 18,2\% equinocandinas, 15,1\% posaconazol y 3,0\% fluconazol. La indicación para el inicio de esta terapia fue la presencia de nódulos en los estudios imagenológicos en 17 episodios (51,5\%), dos pacientes con fungemia $(6,0 \%)$ dos pacientes con niveles elevados de galactomanano $(6,0 \%)$. Los otros pacientes no tenían consignado en la HC claramente la indicación de la terapia.
Respecto a la susceptibilidad bacteriana identificada en los episodios de NF con bacteriemia se encontró: $S$. aureus $3(75 \%)$ eran multisensibles y $1(25 \%)$ resistente a meticilina, ninguno de ellos fue resistente a vancomicina. De las especies gramnegativas aisladas en sangre 29 (74\%) eran sensibles a ciprofloxacina; los 10 casos de resistencia se presentaron en E. coli 8 (80\%), P. aeruginosa 1 (10\%) y un caso en $S$. maltophilia (esta última intrínsecamente resistente).

Hubo resistencia de las especies gramnegativas a piperacilina/tazobactam en $5 / 39(12 \%)$ episodios todos ellos causados por $K$. pneumoniae. La presencia de $\beta$-lactamasas de espectro extendido (BLEE) se detectó en 3/39 (7,6\%) episodios (una cepa de K. pneumoniae y dos de $E$. coli) y sólo hubo un episodio con resistencia a carbapenémicos, correspondiente a la misma K. pneumoniae productora de BLEE.

\section{Mortalidad por episodio}

Durante la estancia hospitalaria fallecieron $8(7,9 \%)$ pacientes de los 101 episodios de neutropenia febril. La mortalidad se atribuyó a la infección en $5(62,5 \%)$ y a progresión de la neoplasia hematológica con resolución de la NF en $3(37,5 \%)$.

La totalidad de los casos que fallecieron tenían bacteriemia, lo cual nos permite calcular la mortalidad total de NF asociada a bacteriemia en nuestra institución de $17 \%(8 / 46)$.

\section{Discusión}

Nuestro estudio es una serie de casos de pacientes con enfermedad hematológica maligna que recibieron algún tipo de quimioterapia y desarrollaron NF requiriendo tratamiento hospitalario. El seguimiento se limitó al tiempo de hospitalización. Existen pocos trabajos en nuestro país que describan las características de esta población ${ }^{15-18}$.

De manera similar a otros estudios, los principales diagnósticos hematológicos fueron LMA, LLA y linfomas $^{1,16,19}$.

La frecuencia de episodios de NF con bacteriemia confirmada fue de $45 \%$, casi el doble de lo referido en la literatura científica de países desarrollados ${ }^{1,20,21}$; pero similar a otros estudios latinoamericanos ${ }^{16,19,22-26}$

La prevalencia de infecciones por microorganismos gramnegativos por sobre grampositivos en los hemocultivos se asemeja a lo comunicado en países desarrollados una veintena antes ${ }^{27}$ y a los datos recientes en estudios latinoamericanos ${ }^{16,19,22}$; pero difiere de los estudios actuales en países del primer mundo donde las cocáceas grampositivas son predominantes ${ }^{1}$.

Llama la atención que el porcentaje de bacilos gramnegativos en nuestro estudio es mucho mayor al referido en otros estudios colombianos y latinoamericanos ya citados 
y adicionalmente la baja frecuencia de aislamiento de Staphylococcus coagulasa negativa, a pesar de que un gran porcentaje de los pacientes tenía accesos vasculares. (Tabla 5). Este segundo hallazgo no es atribuible al uso rutinario de profilaxis con quinolonas en pacientes de alto riesgo, ya que en nuestra institución esta estrategia sólo se utiliza en situaciones de trasplante alogénico de precursores hematopoyéticos. Otros factores que pudieran incidir en la baja frecuencia de $S$. coagulasa negativa son el buen uso de las técnicas de asepsia y antisepsia en el manejo de los catéteres endovasculares y de los protocolos de aislamiento y bioseguridad utilizados en nuestra institución.

Las especies microbianas aisladas con mayor frecuencia (E. coli, K. pneumoniae y $S$. aureus), fueron similares a las encontradas en otras series ${ }^{1,15,19,21,22}$.

Como fortaleza, encontramos que por tratarse de un trabajo observacional donde el investigador no controla las intervenciones realizadas en los pacientes, el estudio refleja lo que ocurre en nuestra práctica diaria.

Como limitantes inherentes al tipo de estudio tenemos varios elementos, en primer lugar sesgos en la selección de pacientes, sesgos de medida y la posibilidad de datos incompletos o no reportados en la historia clínica; particularmente en el año 2009 hubo un sub-registro por cambio en el personal del grupo de hematología. Siendo éste un estudio efectuado en un solo centro, puede sobredimensionarse el predominio de los bacilos gramnegativos en el país, por un comportamiento epidemiológico y microbiológico que puede ser propio de nuestra institución.

En resumen, se presentan los resultados de un estudio con dos años de seguimiento de 101 episodios de NF donde las características microbiológicas difieren significativamente de los países desarrollados, aunque la mortalidad por episodio fue similar.

Un ensayo clínico comparando dos estrategias en la prevención de infecciones asociadas a la neutropenia post-quimioterapia, (profilaxis con quinolonas vs placebo), podría modificar el perfil microbiológico hallado en esta revisión de nuestros pacientes y su desenlace. Sin embargo, teniendo en cuenta la alta resistencia de enterobacterias a quinolonas en nuestro medio ${ }^{28}$, el potencial impacto que puede tener su uso masivo en el desarrollo de resistencia y un aumento del riesgo para adquirir infecciones por $S$. aureus resistente a meticilina ${ }^{29}$ no se considera implementar la quimioprofilaxis con quinolonas dentro de las estrategias de rutina en las guías de manejo de nuestra institución.

Agradecimientos: A la Facultad de Medicina de la Universidad Pontificia Bolivariana y el Hospital Pablo Tobón Uribe, instituciones que ofrecieron al grupo investigador todo el apoyo logístico necesario para llevar a cabo la recolección de datos y elaboración del artículo de investigación pero
Tabla 5. Comparación de los hallazgos microbiológicos entre algunos de los estudios latinoamericanos. HPTU 2009-2011

\begin{tabular}{|c|c|c|c|}
\hline $\begin{array}{l}\text { Estudios } \\
\text { latinoamericanos }\end{array}$ & $\begin{array}{c}\text { Aislamiento } \\
\text { microbiológico (\%) }\end{array}$ & $\begin{array}{c}\text { Bacilos } \\
\text { gramnegativos (\%) }\end{array}$ & $\begin{array}{c}\text { Cocáceas } \\
\text { grampositivas (\%) }\end{array}$ \\
\hline Nuestro estudio & 51,4 & 85,0 & 15,0 \\
\hline Cortés et al ${ }^{15}$ & 35,0 & 36,0 & 52,0 \\
\hline Muñoz et al ${ }^{16}$ & 51,0 & 59,0 & 32,0 \\
\hline Bermúdez et al ${ }^{17}$ & 37,8 & 60,7 & 32,1 \\
\hline Betancur et al ${ }^{18}$ & - & 76,0 & 27,5 \\
\hline Gaytan et al ${ }^{19}$ & 35,0 & 52,0 & 48,0 \\
\hline Bardossy et a ${ }^{22}$ & 35,7 & 52,2 & 43,1 \\
\hline Figuera et $\mathrm{al}^{23}$ & 41,0 & 48,0 & 35,1 \\
\hline Gaytan et al ${ }^{24}$ & 51,0 & 31,0 & 69,0 \\
\hline Puga et $\mathrm{al}^{25}$ & 35,0 & 54,1 & 44,1 \\
\hline Rabagliati et al ${ }^{26}$ & $49^{\prime} 3$ & 51,0 & 41,0 \\
\hline
\end{tabular}

no tuvieron participación alguna en el diseño, recolección, análisis, interpretación de los datos y en la elaboración del manuscrito para publicación. A todas aquellas personas que en una u otra forma colaboraron en la realización del presente trabajo.

\section{Resumen}

Introducción: La neutropenia febril (NF) es un efecto adverso importante post-quimioterapia por su alta morbimortalidad. Hay pocos estudios en nuestro entorno con estos pacientes. Objetivo: Describir las características de los pacientes adultos con neoplasia hematológica que desarrollaron NF post-quimioterapia. Metodología: Estudio descriptivo de serie de casos, en pacientes con neoplasia hematológica y NF post-quimioterapia en el Hospital Pablo Tobón Uribe. Resultados: Ciento un episodios de NF en 43 pacientes con una mediana de edad de 44 años. El $63,5 \%$ no tenían foco infeccioso clínico aparente al ingreso, $11,8 \%$ tenía compromiso de tejidos blandos y $8,9 \%$ foco urinario. Se documentó bacteriemia primaria en $42(41,5 \%)$ y bacteriemia asociada al catéter en $4(3,96 \%)$. Los microorganismos más frecuentes fueron Escherichia coli 43,4\%, Klebsiella pneumoniae 17,3\% y Staphylococcus aureus 8,69\%. De los aislados en sangre, 84,7\% fueron bacilos gramnegativos y $15,2 \%$ cocáceas grampositivas. Piperacilina/tazobactam fue la antibioticoterapia empírica inicial en $81,1 \%$ de los episodios. La mortalidad por episodios de NF fue de $7,92 \%$, en $62,5 \%$ atribuible a la infección y en el resto a progresión de la neoplasia hematológica con resolución de la NF. Conclusión: Serie de casos de NF con características microbiológicas que difieren significativamente a los países desarrollados, pero con una mortalidad por episodios similar. 
Los pacientes con neoplasia hematológica que presentan un episodio de neutropenia febril (NF) deben cumplir con el siguiente protocolo de estudio y manejo.

Una vez que el paciente con un recuento absoluto de neutrófilos $\leq 500$ céls $/ \mathrm{mm}^{3}$ presenta temperatura $\geq 38,3^{\circ}$ en una ocasión o temperatura de $38^{\circ}$ en dos tomas durante una hora se procede a la obtención de dos hemocultivos por vena periférica y, en caso de que el paciente tenga catéter venoso central, un hemocultivo por cada luz del dispositivo. A todos los pacientes se les solicita radiografía de tórax y se toman muestras para citoquímico de orina y urocultivo; pruebas de función hepática, función renal y muestra de cualquier foco probable de infección: tinción de Gram y cultivo de esputo si existen síntomas y signos respiratorios, coprocultivo y leucocitos fecales si presenta diarrea, aspirado y biopsia de piel en caso de lesiones en piel, punción articular, toracocentesis o paracentesis con estudios de líquidos, según el caso en particular.

Una vez hecho el diagnóstico de NF se inicia tratamiento antimicrobiano empírico con piperacilina/tazobactam; está indicado iniciar fluconazol en caso de que el paciente presente mucositis. Cumplidas $72 \mathrm{~h}$ del esquema inicial, si el paciente continúa con fiebre o desarrolla fiebre a partir de entonces, y no presenta compromiso hemodinámico, se debe efectuar una re-evaluación clínica y de laboratorio incluyendo medición seriada de galactomanano.

La prescripción de vancomicina se reserva para aquellos pacientes que presentan mucositis grave post-quimioterapia con ara C y 5 FU, sospecha de infección asociada a catéter o tejidos blandos, el aislamiento de una bacteria grampositiva en identificación y pacientes en estado de shock. Si al tercer día de antibioticoterapia los hemocultivos obtenidos al ingreso son negativos, se suspende la vancomicina.

En caso de sospecha clínica de aspergilosis se inicia voriconazol.

Finalmente, sólo se utiliza antibioticoterapia profiláctica con ciprofloxacina en pacientes en pacientes con quimioterapia para trasplante alogénico.

\section{Referencias bibliográficas}

1.- Klastersky J, Ameye L, Maertens J, Georgala A, Muanza F, Aoun M, et al. Bacteraemia in febrile neutropenic cancer patients.Int J Antimicrob Agents 2007; 30 Suppl 1: S51-9.

2.- Gafter-Gvili A, Fraser A, Paul M, Vidal L, Lawrie T A, van de Wetering M D, et al. Antibiotic prophylaxis for bacterial infections in afebrile neutropenic patients following chemotherapy. Cochrane Database Syst Rev 2012; 1: CD004386.

3.- Bucaneve G, Micozzi A, Menichetti F, Martino P, Dionisi MS, Martinelli G, et al. Levofloxacin to prevent bacterial infection in patients with cancer and neutropenia. N Engl J Med 2005; 353(10): 977-87.

4.- Bucaneve G, Castagnola E, Viscoli C, Leibovici L, Menichetti F. Quinolone prophylaxis for bacterial infections in afebrile high risk neutropenic patients. Eur J Cancer 2007; 5 (2): 5-12.

5.- Pizzo P A, Robichaud K J, Gill F A, Witebsky F G. Empiric antibiotic and antifungal therapy for cancer patients with prolonged fever and granulocytopenia. Am J Med 1982; 72 (1): 101-11.

6.- Schimpff S C. Empiric antibiotic therapy for granulocytopenic cancer patients. Am J Med 1986; 80 (5C): 13-20.

7.- Schimpff S, Satterlee W, Young V M, Serpick A. Empiric therapy with carbenicillin and gentamicin for febrile patients with cancer and granulocytopenia. N Engl J Med 1971; 284 (19): 1061-5.

8.- Freifeld A G, Bow E J, Sepkowitz K A, Boeckh M J, Ito J I, Mullen C A, et al. Clinical practice guideline for the use of antimicrobial agents in neutropenic patients with cancer: 2010 update by the Infectious Diseases Society of America. Clin Infect Dis 2011; 52(4): e56-93.

9.- Glasmacher A, von Lilienfeld-Toal M, Schulte S, Hahn C, Schmidt-Wolf I G H, Prentice A. An evidence-based evaluation of important aspects of empirical antibiotic therapy in febrile neutropenic patients. Clin Microbiol Infect 2005; 11 Suppl 5: 17-23.

10.- Paul M, Yahav D, Bivas A, Fraser A, Leibovici L. Anti-pseudomonal beta-lactams for the initial, empirical, treatment of febrile neutropenia: comparison of beta-lactams. Cochrane Database Syst Rev 2010; (11): CD005197.

11.- Food and Drug Administration (US). Cefepime (marketed as Maxipime) (Internet). Silver Spring, MD: Food and Drug Administration; 2009 (citado 2012 jun 14). Available in: http:// www.fda.gov/Drugs/DrugSafety/Postmarket DrugSafetyInformationforPatientsandProviders/ DrugSafetyInformationforHeathcare Professionals/ucm167254.htm

12.- National Comprehensive Cancer Network. Prevention and treatment of cancer: related infections (Internet). Washington, PA: National Comprehensive Cancer Network; 2009 (citado 2012 abr 1). Available in: oralcancerfoundation. org/treatment/pdf/infections-NCCN.pdf

13.- Mermel L A, Allon M, Bouza E, Craven D E, Flynn P, O'Grady N P, et al. Clinical practice guidelines for the diagnosis and management of intravascular catheter-related infection: 2009 Update by the Infectious Diseases Society of America. Clin Infect Dis 2009; 49 (1): 1-45.

14.- Dekkers O M, Egger M, Altman D G, Vandenbroucke J P. Distinguishing case series from cohort studies. Ann Intern Med 2012; 156 (1 Pt 1): 37-40.

15.- Cortés J, Cuervo S, Arroyo P, Quevedo R.
Hallazgos microbiológicos en pacientes con neutropenia febril. Rev Colomb Cancerol 2003; 7 (4): 5-11.

16.- Muñoz Maya O G, Rodelo Vélez A M, Carvajal J J, González J M, Jaimes Barragán F A. Características clínicas y microbiológicas de los pacientes neutropénicos febriles con neoplasias hematológicas. Iatreia. 2008; 21(1): S9.

17.- Bermúdez C D. Caracterización de la neutropenia febril en pacientes con leucemia linfoide aguda, tratados con quimioterapia de alto riesgo, atendidos en el Instituto Nacional de Cancerología desde 1 de enero al 31 de diciembre de 2008 (Internet). Bogotá: Universidad Nacional de Colombia; 2009 (citado 2012 jun 13). Available in: www. bdigital.unal.edu.co/2447/1/598245.2009.pdf

18.- Betancur C A, Lema M, Arcila G. Etiologic agents in 54 consecutive febrile neutropenia $(\mathrm{FN})$ isolates in a general hospital in Medellín, Colombia. J Clin Oncol 2012; 30 (Suppl): Abstr e19604.

19.- Gaytán-Martínez J, Mateos-García E, Sánchez-Cortés E, González-Llaven J, Casanova-Cardiel L J, Fuentes-Allen J L. Microbiological findings in febrile neutropenia. ArchMed Res 2000; 31 (4): 388-92.

20.- Viscoli C, Cometta A, Kern W V, Bock R, Paesmans M, Crokaert F, et al. Piperacillintazobactam monotherapy in high-risk febrile and neutropenic cancer patients. Clin Microbiol Infect 2006; 12 (3): 212-6.

21.- Sigurdardottir K, Digranes A, Harthug S, Nesthus I, Tangen J-M, Dybdahl B, et al. A multi-centre prospective study of febrile neutropenia in Norway: microbiological findings and antimicrobial susceptibility. Scand J Infect Dis 2005; 37 (6-7): 455-64. 
22.- Bardossy A, Petiti H, Safar N, Zlocowsk J, Zárate A. Neutropenia febril: agentes etiológicos y respuesta a antibiótico terapia empírica en el Hospital Privado S.A. Centro Médico de Córdoba durante 2006-2007. Exp Médica 2011; 29 (3): 5-15.

23.- Figuera Esparza M, Carballo M, Silva M, Figueredo A, Avilán J. Microbiological isolates in patients with febrile neutropenia and hematological neoplasias. Rev Esp Quimioter 2006; 19 (3): 247-51.

24.- Gaytán-Martínez J, Avila-Morán M, Mata-Marín J A, Mateos-García E, Fuentes-Allen J L, Vela-Ojeda J, et al. Prevalence of bacterial infections and patterns of bacterial susceptibility among patients with fever, neutropenia, and hematological neoplasias.Gac Med Mex 2011; 147 (4): 325-32.

25.- Puga L B, Puga R I, Cabrera-C M E, Undurraga-S M S, Guerra-C C, Urrejola-S G, et al. Neutropenia febril de alto riesgo en leucemia aguda. Experiencia en un hospital público: Programa Nacional de Drogas Antineoplásicas del Adulto (PANDA), Hospital del Salvador, 1991-2001. Rev Med Chile 2003; 131 (9): 1023-30.

26.- Rabagliati-B R, Fuentes-L G, Orellana-U E, Oporto-C J, Domínguez-M I, Benítez-G R, et al. Etiology of febrile neutropenia episodes among cancer patients from Hospital Clínico Universidad Católica, Santiago-Chile. Rev
Chilena Infectol 2009; 26 (2): 106-13.

27.- Zinner SH. Changing epidemiology of infections in patients with neutropenia and cancer: emphasis on gram-positive and resistant bacteria. Clin Infect Dis 1999; 29 (3): 490-4.

28.- GERMEN. Escherichia coli. Perfiles de sensibilidad a antibióticos de Escherichia coli en Servicios NO UCI GERMEN 2009-20102011 [Internet].Medellín: GERMEN; 2012 [citado 2012 may 31]. Available in: http://www. grupogermen.org/

29.- Rangaraj G, Granwehr B P, Jiang Y, Hachem R, Raad I. Perils of quinolone exposure in cancer patients: breakthrough bacteremia with multidrug-resistant organisms. Cancer 2010; 116 (4): 967-73. 